\title{
Evolution of Better Wavelet Coefficients for Fingerprint Image Compression using cropped images
}

\author{
K.T.Shanavaz, P.Mythili \\ Division of Electronics, School of Engineering \\ Cochin University of Science \&Technology \\ Kochi, Kerala, India \\ shanavazkt@cusat.ac.in,mythili@cusat.ac.in
}

\begin{abstract}
This paper explains the Genetic Algorithm (GA) evolution of optimized wavelet that surpass the cdf $9 / 7$ wavelet for fingerprint compression and reconstruction. Optimized wavelets have already been evolved in previous works in the literature, but they are highly computationally complex and time consuming. Therefore, in this work, a simple approach is made to reduce the computational complexity of the evolution algorithm. A training image set comprised of three $32 \times 32$ size cropped images performed much better than the reported coefficients in literature. An average improvement of $1.0059 \mathrm{~dB}$ in PSNR above the classical cdf9/7 wavelet over the 80 fingerprint images was achieved. In addition, the computational speed was increased by $90.18 \%$. The evolved coefficients for compression ratio (CR) 16:1 yielded better average PSNR for other CRs also. Improvement in average PSNR was experienced for degraded and noisy images as well.
\end{abstract}

Keywords- wavelet; lifting scheme; evolved transforms; genetic algorithms; image compression; fingerprint

\section{INTRODUCTION}

Image compression is intended to get the best possible image quality at an allowed storage facility. It is required for easy storage of images and their faster transmission over band-limited communication channels. For this FBI fingerprint identification division has took on Wavelet Scalar Quantization (WSQ) as standard for fingerprint image compression [1]. Forensic, medical applications, law enforcement and border security are some vital areas where fingerprint image compression finds a significant role.

Wavelet based image compression [2,3] is capable of examining the image signal at different resolutions. This is termed as multiresolution analysis (MRA). DWT is an algorithm that transforms the original image to horizontal, vertical and diagonal decomposition coefficients. Biorthogonal wavelets [4] show both symmetry and compact support. Several wavelets and image compression techniques have been described in literature [5,2,6,7,8] In 1992, Cohen, Daubechies, and Feauveau (cdf) introduced the classical cdf9/7 biorthogonal wavelet filters [9], which is used by the FBI fingerprint compression standard [10,11].

The classical cdf 9/7 DWT is represented by four sets of filter coefficients. Altogether there are 16 coefficients [11]. Peak-Signal-to-Noise-Ratio (PSNR) and Root Mean Squared Error (RMSE) are the two measures of image compression performance [12]. The lifting scheme introduced by $\mathrm{W}$. Sweldens [7, 13, 14] is an effective technique to represent classical wavelets with lesser number of coefficients. Any DWT or two band sub-band filtering with finite filters could be decomposed into a finite sequence of simple lifting steps. This was performed by factorizing the polyphase matrix of the wavelet or sub-band filters into elementary matrices [14].

Recently several researchers have evolved wavelet filter coefficients using Genetic algorithm (GA) [15, 16]. Brendan Babb evolved classical wavelet filter coefficients [11] for four level MRA. The population size was 240-280 chromosomes and the number of generations was more than 15000. The amount of computational complexity was very high and hence supercomputers were used for evolving coefficients. An average PSNR improvement of $0.76 \mathrm{~dB}$ over 80 fingerprint images in the database was reported. Uli Grasemann and Risto Mikkulainen [17] employed the coevolutionary algorithm and properties of lifting by which specialized wavelets were evolved for fingerprint image compression. Coefficients taken randomly from a Gaussian distribution were used for initial population. The algorithm was too slow as it took 60 hours on a $3 \mathrm{GHz}$ Xeon Processor. An average PSNR improvement of $0.75 \mathrm{~dB}$ was reported. Shanavaz and Mythili [12] proposed a method by which cdf9/7 lifting coefficients were evolved using GA. The population size was 250 and number of generations was 1000. On an AMD Athlon 2.39GHz Processor with $750 \mathrm{MB}$ memory, the algorithm took 33 hours. Over $35.9119 \mathrm{~dB}$ average PSNR obtained by the classical cdf9/7 wavelet, an improvement of $0.9306 \mathrm{~dB}$ could be achieved.

In order to have a fair comparison with the previous results and a speedy evolution of the lifting coefficients, the symmetric lifting scheme $[4,12]$ with only 4 coefficients has been used in this work. Better lifting coefficients have been evolved in a shorter time with training sets comprised of cropped fingerprint images. Thus the computational complexity and time consumption of the evolution procedure have been reduced. The results have been compared with the existing results in literature.

In the rest of this paper, Section II describes the methodology of the proposed work of evolving lifting coefficients and the development of training image sets for fingerprint image compression using GA. Results and discussions are provided in Section III and Section IV concludes the paper. 


\section{Methodology}

\section{A. Evolving Lifting Coefficients}

In this work, symmetrical lifting coefficients of cdf9/7 wavelet have been evolved using GA [4, 12]. The classical cdf 9/7 wavelet system consists of 16 coefficients, whereas the symmetrical lifting system consist only 4 coefficients. Faster evolution is possible with the lesser number of lifting coefficients. Consequently, the evolved coefficients compress the fingerprint images within a shorter time. The block diagram of GA algorithm to evolve optimal cdf 9/7 lifting coefficients for fingerprint image compression is shown in Fig. 1. Binary GA is used for the evolution [12].

The initial population of chromosomes is comprised of mutated copies of lifting coefficients. These coefficients are used to transform the training images into the wavelet realm. As in the previous works in literature, the compression ratio (CR) is fixed to $16: 1$. For this, the smallest $93.75 \%$ of wavelet transformed image coefficients are set to zero for image reconstruction and the others are retained. PSNR, the fitness function of the GA, is computed to evaluate the quality of the reconstructed image/s.

A 17 bit binary number is used to represent each coefficient. The first bit corresponds to sign and the other bits correspond to the coefficient value. A binary chromosome is formed by a group of 4 coefficients. Initial GA population is formed from randomly mutated copies of the symmetrical lifting coefficients of cdf9/7. The population is formed by 250 numbers of chromosomes. The number of generations is 1000 . Roulette wheel selection and single point crossover are employed. Crossover rate $=0.7$, mutation rate $=0.0075$ and elitism $=1$. The algorithm was implemented in Matlab.

The chromosomes are arranged according to their fitness. Then, the best are participated in reproduction. To produce new set of chromosomes, these chromosomes are subjected to crossover and mutation operations. An

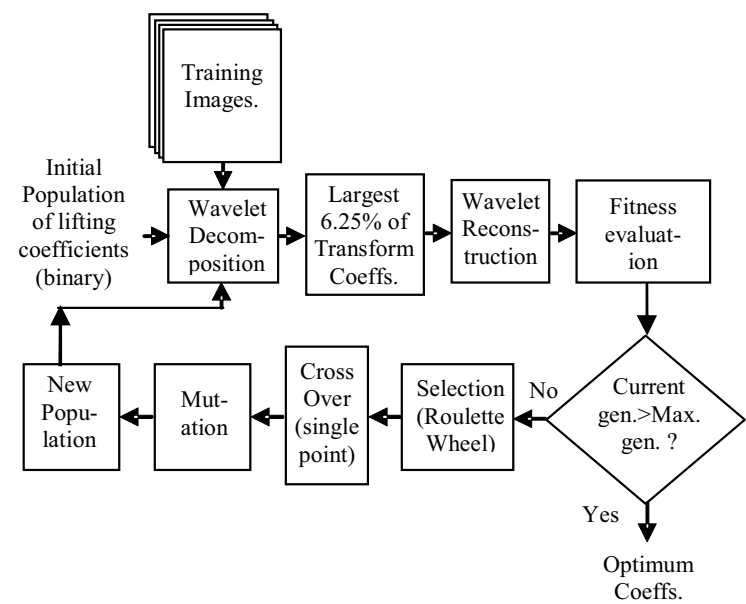

Figure 1. Block diagram of the evolutionary algorithm (GA) for optimizing cdf9/7 lifting coefficients for Fingerprint image compression. optimum set of coefficients is evolved by reiterating the above operations with the new populations. For the selected training image set, the best PSNR is obtained for the best set of evolved coefficients. Finally, these best set of coefficients are used to compute the average improvement in PSNR over 80 fingerprint images in the database.

\section{B. Formation of training image set}

The image set $\mathrm{B}$ of the DB1 database in Fingerprint Verification Competition (FVC) 2000 database contains 80 fingerprint images. Each image has a size $300 \times 300$ pixels 500 dpi resolution. The training image set has been developed from this image set.

In the earlier works $[11,12,17]$ the training image set contained four numbers of full-size $(300 \times 300$ pixels) fingerprint images. In the present work, to speed up the evolution process, training image set was formed by images cropped to smaller sizes. To begin with, the training image sets consisting of image/s cropped to $8 \times 8$ pixels size were created. Starting with one cropped image of $8 \times 8$ pixels size, images of the same size were added in the training set one by one up to 10 . It was found that the evolved coefficients gave reduced PSNR compared to the theoretical lifting coefficients. A similar observation was made in the case of training sets comprising of cropped images with 16x16 pixels size too. Next, the training sets built from image/s cropped to $32 \times 32$ pixels size were used and the performances of the evolved coefficients were studied. A typical full-size (300x300 pixels) fingerprint image and the image cropped to $32 \times 32$ pixels are shown in Fig. 2.

\section{ReSUlTS AND Discussions}

\section{A. Improvement in PSNR with the evolved coefficients}

The different training image sets with suitable number of images of $32 \times 32$ pixels size to give maximum PSNR were constructed as described in the previous section. Optimum coefficients providing maximum PSNR for CR 16:1 were evolved with these sets. Using these coefficients, average

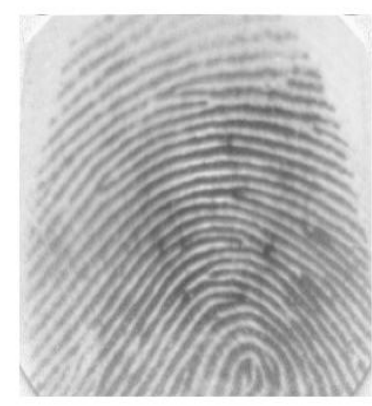

(a) (b)
Figure 2. Block diagram of the evolutionary algorithm (GA) for optimizing cdf9/7 lifting coefficients for FIC. 
improvement in PSNR over classical [18, 19] cdf9/7 wavelet for various nos. and sizes of training images were computed.

In order to perform a comparison, the previous [12] and the present algorithms must be run on the same hardware platform. So, both he algorithms were run on an Intel Xeon $3.00 \mathrm{GHz}$ Processor with $6 \mathrm{~GB}$ memory. In this platform the previous algorithm [12] took an evolution time of 9.06 hours whereas the proposed work took only $0.89 \mathrm{hrs}$. This amounts to an increase in computational speed by $90.18 \%$. A maximum improvement of $1.0059 \mathrm{~dB}$ in average PSNR above the classical cdf9/7 wavelet over the 80 fingerprint images was obtained with training image set comprising of three $32 \times 32$ size images. The evolution-time and PSNR are better than the previous results $[17,20,21,12]$.

Plot of PSNR for classical and evolved wavelet is shown in Fig. 3. For all the 80 images, the performance of the evolved wavelet is much better than that of the classical wavelet. The original fingerprint image 101_1.tif and the image reconstructed using classical and evolved cdf 9/7 lifting coefficients for compression ratio $16: 1$ are shown in Fig. 4.

\section{B. Improvement in PSNR for other CRs}

The evolved coefficients show PSNR improvement for various other CRs too. The PSNRs of the evolved and classical wavelets for various CRs are compared in Fig. 5. The evolved wavelets provided better PSNR over the classical wavelet, for all values of CRs.

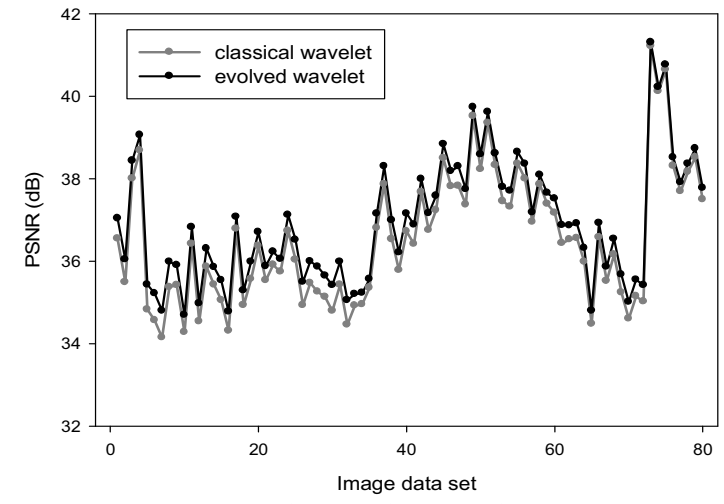

Figure 3. PSNR for classical and evolved wavelets for $\mathrm{CR}=16: 1$.

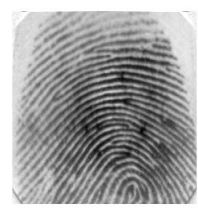

(a)

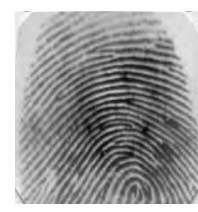

(b)

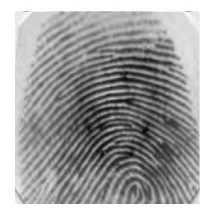

(c)
Figure 4. 101_1.tif fingerprint image (a) original, reonstructed image using classical (35.91 dB) ( (c) evolved wavelets $(37.04 \mathrm{~dB})$ for $\mathrm{CR}=16: 1$.

\section{Improvement in PSNR of degraded images}

Quality of the input images were degraded to different quantities by setting certain percentages of lower pixel values to zero. Percentage degradation was computed using the following eqn. [12].

$$
\text { Degradation }=\frac{\text { No. of pixels set to zero }}{\text { Total no. of pixels }} \times 100 \% \text {. }
$$

Fig. 6 shows the comparison of average PSNRs of degraded images for $\mathrm{CR}=10: 1$, for classical and evolved wavelets. The PSNR corresponding to the classical wavelet was better than the evolved PSNR for lower values of degradation. The evolved PSNR became well again beyond $42 \%$ of degradation.

\section{PSNRs of noisy images}

Fig. 7 illustrates the comparison of average PSNRs of noisy images for $\mathrm{CR}=16: 1$, computed from classical and evolved wavelets. Here, Gaussian white noise with mean 0 and variance 0.05 was added to the image. The noisy image (101 1.tif) is shown in Fig. 8. The images reconstructed with classical and evolved wavelets are also shown. The PSNRs of the noisy image owing to evolved wavelet were better for higher CRs. For lower CRs PSNR values are almost equal.

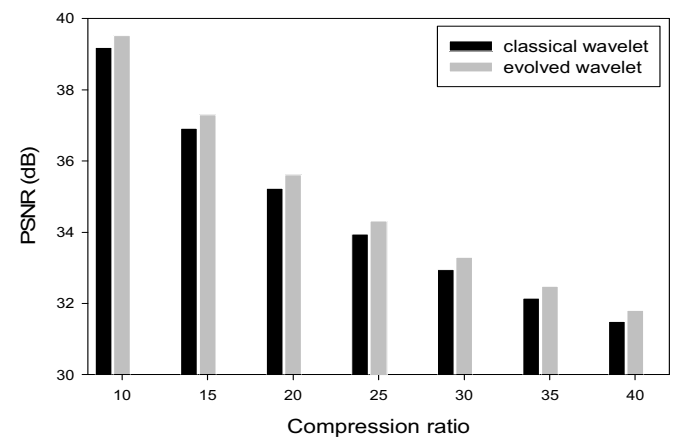

Figure 5. Plot of average PSNRs for classical and evolved wavelets for $\mathrm{CR}=16: 1$.

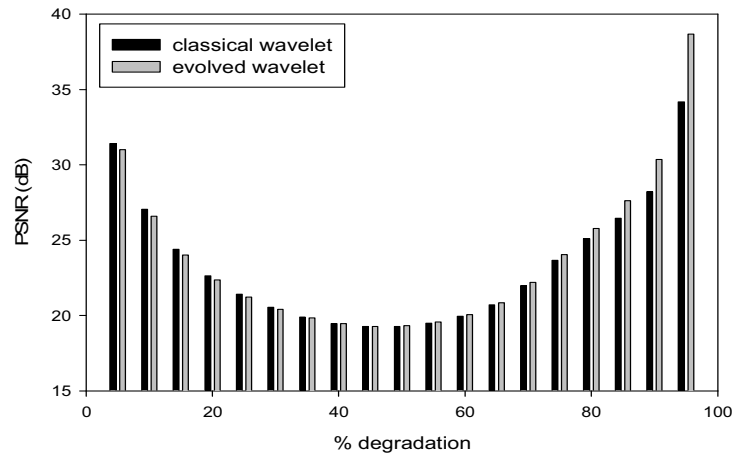

Figure 6. Comparison of average PSNRs of degraded images for $\mathrm{CR}=10: 1$, computed from classical and evolved wavelets. 


\section{CONCLUSION}

The proposed work describes techniques for evolving better wavelet coefficients for compression and reconstruction of fingerprint images with improved quality. Here, lifting scheme and GA are combined and used to evolve better lifting filter coefficients of cdf9/7 wavelet.

The evolution process for optimizing wavelets, described in previous works in literature were highly computationally complex and time consuming. So, in this work, in order to reduce the computational complexity and evolution time, small cropped portions of the fingerprint images are taken as training image set. The training image set comprising of three $32 \times 32$ size cropped images performed much better than the reported coefficients in literature. A maximum improvement of $1.0059 \mathrm{~dB}$ in average PSNR above the classical cdf $9 / 7$ wavelet over the 80 fingerprint images was obtained. In addition, the computational speed was increased by $90.18 \%$. The evolved coefficients for CR 16:1 yielded better average PSNR for other CRs also. Improvement in average PSNR was experienced for degraded and noisy images too. For specific class of images, compression performance can be improved by designing proper training image set.

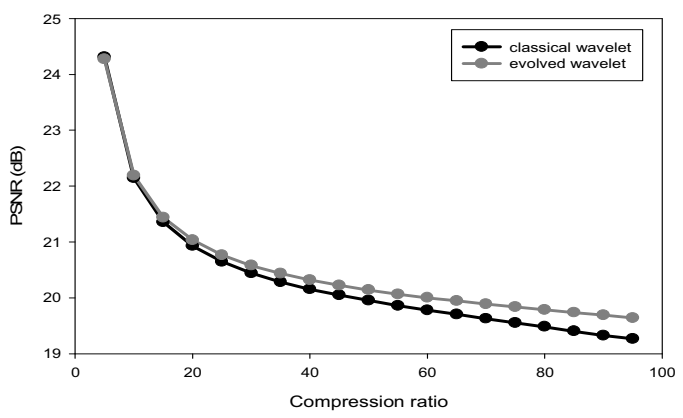

Figure 7. Comparison of PSNRs of noisy image (101 1.tif) for various CRs, computed from classical and evolved wavelets.

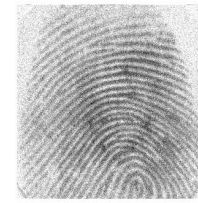

(a)

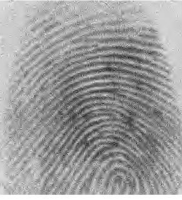

(b)

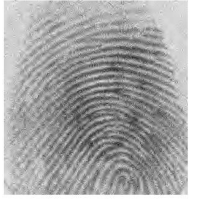

(c)
Figure 8. (a) Noisy fingerprint image (101_1.tif) (b) reonstructed image using classical $(21.30 \mathrm{~dB})(\mathrm{c})$ evolved wavelets $(21.40 \mathrm{~dB})$ for $\mathrm{CR}=16: 1$.

\section{REFERENCES}

[1] D.Maltoni, D.Maio, A.K.Jain, S.Prabhakar, Handbook of Fingerprint Recognition, $2^{\text {nd }}$ ed., Springer, 2009.
[2] I. Daubechies, Ten Lectures on Wavelets, SIAM, 1992.

[3] A.S. Lewis, and G. Knowles, "Image compression using the 2D wavelet transform”, IEEE Trans. on Im. Proc., vol. 1, 244-250, 1992.

[4] D. Taubman, and M Marcellin, JPEG2000: Image Compression Fundamentals, Standards, and Practice, Kluwer Academic Publishers, Boston/Dordrecht/London,2002.

[5] S. Mallat, "A theory for multiresolution signal decomposition: the wavelet representation", IEEE Trans. on Patt. Anal. and Mach. Intelligence, vol. 11, 674-693, 1989.

[6] J.M. Shapiro, "Embedded image coding using zerotrees of wavelet coefficients", IEEE Trans. on Sig. Proc., vol.41, 3445-3462, 1993.

[7] W. Sweldens, "The Lifting Scheme: A custom-design construction of biorthogonal wavelets", J. of Applied and Computational Harmonic Analysis, vol. 3(2), 186-200, 1996.

[8] A. Said, and W.A. Pearlman, "A new, fast and efficient image codec based on set partitioning in hierarchical trees", IEEE Trans. on Circuits and Syst. for Video Technology, vol.6, 243-250, June 1996.

[9] A. Cohen, I. Daubechies, and J.-C. Feauveau "Biorthogonal Bases of Compactly Supported Wavelets," Communications on Pure and Applied Mathematics, 45 (5), 485-560, June 1992.

[10] J. Bradley, C. Brislawn, and T. Hopper, "The FBI Wavelet/ Scalar Quantization Standard for Gray-Scale Fingerprint image Compression," SPIE Vol. 1961, Visual Information Processing II (1993): 293-304.

[11] B. Babb, "Evolved transforms surpass the FBI wavelet for improved fingerprint compression and reconstruction," Proc. GECCO conf. companion on Genetic and evolutionary computation, London, July 7-11, 2007.

[12] K.T.Shanavaz, P.Mythili, "An Improved Technique for Evolving Wavelet Coefficients for Fingerprint Image Compression", Proc. IEEE Int. Conf. on Commn. Control and Computing Techs. (ICCCCT 2010), Ramanathapuram,India, October 2010, 665-669.

[13] W. Sweldens, "The Lifting Scheme: A construction of secondgeneration wavelets," SIAM J.Math Anal., 29 (1997) 511-546.

[14] I. Daubechies, W. Sweldens, "Factoring wavelet transforms into lifting steps," J. of Fourier Anal. and Appl., 4 (1998), 245-267.

[15] D. Goldberg, Genetic Algorithms in Search, Optimization, and Machine, $1^{\text {st }}$ ed., Addison- Wesley, 1989.

[16] S.N.Sivanandan, .S.N.Deepa, Introduction to Genetic Algorithms, Springer,2008.

[17] U. Grasemann, and R. Mikkulainen, "Effective Image Compression using Evolved Wavelets," Proc. of the 7th Annual Genetic and Evolutionary Comp. Conf. (GECCO’2005) , 6/25-29, 2005, Washington, DC, 2: 1961-1968, ACM.

[18] J. Villasenor, B. Belzer, and J. Lia, "Wavelet filter evaluation for image compression", IEEE Trans. on Im. Proc., vol.2, 1053-1060, 1995.

[19] G. Davis, and A. Nosratinia, "Wavelet-based image coding: An overview", in Appl. and Comp. Control, Signals and Circuits, ed. Biswa Nath Datta, Boston, NY, USA: springer-verlag.

[20] B. Babb, and F. Moore, 'The best fingerprint compression standard yet', in Systems, Man and Cybernetics, IEEE Int. Conf., 2911-2916, 2007.

[21] B. Babb, F. Moore, and M. Peterson, "Improved multiresolution analysis transforms for satellite image compression and reconstruction using evolution strategies", in Proc. of the 11th Annual Conf. Companion on Genetic and Evolutionary Computation , ACM, 2547-2552, 2009. 